NBER WORKING PAPER SERIES

\title{
THE PERFORMANCE OF THE PIVOTAL-VOTER MODEL IN SMALL-SCALE ELECTIONS: EVIDENCE FROM TEXAS LIQUOR REFERENDA
}

\author{
Stephen Coate \\ Michael Conlin \\ Andrea Moro \\ Working Paper 10797 \\ http://www.nber.org/papers/w10797
NATIONAL BUREAU OF ECONOMIC RESEARCH
1050 Massachusetts Avenue
Cambridge, MA 02138
September 2004

We thank Bill Goffe, Sam Kortum, Stephen Ross, and Birali Runesha for their comments and help. We acknowledge support from the Minnesota Supercomputing Institute. The views expressed herein are those of the authors and not necessarily those of the Federal Reserve Bank of Minneapolis, the Federal Reserve System or the National Bureau of Economic Research.

(C2004 by Stephen Coate, Michael Conlin, and Andrea Moro. All rights reserved. Short sections of text, not to exceed two paragraphs, may be quoted without explicit permission provided that full credit, including () notice, is given to the source. 
The Performance of the Pivotal-Voter Model in Small-Scale Elections: Evidence from Texas Liquor Referenda

Stephen Coate, Michael Conlin, and Andrea Moro

NBER Working Paper No. 10797

September 2004

JEL No. D7

\title{
ABSTRACT
}

How well does the pivotal-voter model explain voter participation in small-scale elections? This paper explores this question using data from Texas liquor referenda. It first structurally estimates the parameters of a pivotal-voter model using the Texas data. It then uses the estimates to evaluate both the within and out-of-sample performance of the model. The analysis shows that the model is capable of predicting turnout in the data fairly well, but tends, on average, to predict closer electoral outcomes than are observed in the data. This difficulty allows the pivotal-voter model to be outperformed by a simple alternative model based on the idea of expressive voting.

\author{
Stephen Coate \\ Department of Economics \\ Cornell University \\ Ithaca, NY 14853 \\ and NBER \\ sc163@cornell.edu \\ Michael Conlin \\ Department of Economics \\ Syracuse University \\ Syracuse, NY 13244 \\ meconlin@maxwell.syr.edu \\ Andrea Moro \\ Department of Economics \\ University of Minnesota \\ Minneapolis, MN 55455 \\ amoro@atlas.socsci.umn.edu
}




\section{Introduction}

The purpose of this paper is to shed light on the ability of the pivotal-voter model to explain turnout in small-scale elections. According to this model, citizens are motivated to vote by the chance that they might swing the election. Citizens are assumed to rationally anticipate the probability that their votes will be pivotal and to vote if the expected "instrumental benefit" outweighs the cost of going to the polls. A positive level of turnout is assured in equilibrium, since if no citizen were expected to vote, any deviator would be pivotal with probability one.

The pivotal-voter model, as developed by Ledyard (1984) and Palfrey and Rosenthal (1983), (1985), forms the basic framework for thinking about turnout in theoretical political science. It is in many respects the simplest and most natural way of thinking about the problem. It requires only that citizens be instrumentally motivated and that they have rational expectations, core assumptions of rational choice theory. It is relatively tractable and yields a number of interesting implications. ${ }^{1}$

Despite its theoretical appeal, it seems widely accepted that the pivotal-voter model does not provide an empirically satisfactory theory of turnout in large-scale, single-issue elections (see, for example, Feddersen (2004) and Green and Shapiro (1994)). Palfrey and Rosenthal (1985) showed that when voters are uncertain about the preferences of their fellows, the critical cost levels below which citizens vote converge to zero as the number of citizens approaches infinity. Thus, in order for observed outcomes to be consistent with the model, the costs of voting for a large group of citizens must be minuscule. Not only does this seem unlikely, but if it were the case, then it is not clear how to explain the variation in turnout in large elections observed in the data.

Palfrey and Rosenthal's result does not, however, rule out the possibility that the pivotal-voter model provides a reasonable theory for understanding small-scale elections and this seems to be the implicit justification for its continued use in

\footnotetext{
1 For example, Borgers (2004) uses the model to show that when citizens are ex-ante identical, compelling citizens to vote is never desirable on welfare grounds (see also Ghosal and Lockwood (2003)). Campbell (1999) uses the model to show how a policy outcome preferred by a small minority of the electorate can be implemented under majority voting if the preferences of that minority are strong or their costs of voting are low.
} 
theoretical work (see, for example, Borgers (2004)). With a relative small number of voters (e.g., less than 5,000), the equilibrium probability of being pivotal is large enough to motivate voters with positive costs of voting to participate. While it would be cleaner to have a single model to explain turnout in all elections, there is no obvious reason to believe that the forces driving turnout in small-scale elections should be the same as those in large, single-issue elections. Moreover, the empirical relevance of small-scale elections is perhaps greater than is often assumed, because voters in national elections are often simultaneously voting on a host of local issues where the number of voters is small.

To study the performance of the pivotal-voter model in small-scale elections, we use the data set on Texas liquor referenda assembled by Coate and Conlin (2004). The jurisdictions holding these elections are often very small, many having less than 1,000 eligible voters. These elections also have the advantage that they are typically held separately from other elections, so that the only reason to go to the polls is to vote on the proposed change in liquor law. Moreover, the issues decided by the referenda are very similar across jurisdictions.

The paper begins by developing and structurally estimating a parameterized version of the pivotal-voter model. The estimation is a computationally difficult undertaking, given the complexity of the equilibrium conditions implied by the pivotalvoter model. ${ }^{2}$ Because of computational constraints, the parameters of the model are estimated using only those elections in which the number of voting age citizens is less than 900. We then explore how well the pivotal-voter model explains both total turnout and the closeness of the referendum outcomes in these smaller jurisdictions (i.e., "within-sample"). ${ }^{3}$ We find that the model does well predicting total turnout but predicts much closer elections than are seen in the data. We also use our coefficient estimates to compute the equilibrium of the model in the larger jurisdictions (between 906 and 81,904 eligible voters). For these "out-of-sample" observations,

\footnotetext{
2 Evaluating the expected benefit of voting requires computing the equilibrium probability that a voter will be pivotal.

3 We define total turnout as the percent of eligible voters (i.e., voting age population) that turn out to vote. We define closeness as the difference between turnout for and turnout against the proposed change (i.e. the percent of eligible voters that vote for minus the percent of eligible voters that vote against).
} 
the pivotal-voter model underpredicts total turnout.

Finally, we compare the within-sample performance of the pivotal-voter model with that of a simple alternative model based on the idea of expressive voting. This model - the intensity model - simply assumes that citizens vote to express their preferences and that their expressive payoffs are higher the more intensely they feel about an issue. We find that this very simple view of voting explains the election outcomes better than the considerably more sophisticated pivotal-voter model.

To date, despite its popularity in theoretical work, there has been very little research directly testing the performance of the pivotal-voter model. This reflects both the difficulty of finding appropriate data and the analytical difficulties of implementing the model. Indeed, to our knowledge, the only other paper to have even attempted the task is Hansen, Palfrey and Rosenthal (1987). They use data on school budget referenda and make strong assumptions to undertake the estimation. In particular, they assume that the population is equally divided between supporters and opposers of the proposed budget and that both sides have identical benefits from their preferred outcomes. Consistent with these simplifying assumptions, Hansen, Palfrey and Rosenthal estimate the parameters of the model using only referenda with "close" outcomes and focus only on total turnout. As we do, they find that the model estimates match observed levels of turnout reasonably well. However, our analysis imposes neither the equal division nor the identical benefits assumption, which allows us to estimate the parameters of the model using all election outcomes (close and non-close). This permits us to analyze the model predictions regarding not only total turnout but also closeness.

Our paper complements the recent work of Coate and Conlin (2004). Using the same data set, Coate and Conlin structurally estimate the parameters of a novel model of voter turnout which assumes that individuals are motivated to vote by the ethical desire to do their part to help their side win. ${ }^{4}$ They show that this group rule-utilitarian model fits the data well and performs better than the simple intensity model of voter turnout discussed above. While this is certainly interesting, it is natural to wonder how the more standard pivotal-voter model would fare in this

\footnotetext{
4 This model is based on the ideas of Harsanyi (1980) and Feddersen and Sandroni (2002).
} 
environment and this is the issue we take up here. Unfortunately, we are unable to provide direct comparisons of all three models because Coate and Conlin's analysis makes the simplifying assumption of a continuum of voters. This must be dispensed with here because it implies that no voter can be pivotal. However, the intensity model is sufficiently simple that it can be easily estimated with either a finite or a continuum of voters, so we are able to compare its performance with that of the pivotal-voter model. ${ }^{5}$

The organization of the remainder of the paper is as follows. The next section presents our parameterized version of the pivotal-voter model. Section 3 describes the institutional details concerning the referenda that we study and the data. Section 4 explains our estimation strategy and Section 5 describes the results. Section 6 introduces the intensity model and compares its performance with that of the pivotal-voter model. Section 7 summarizes the results and discusses their implications for future research on voter turnout.

\section{The pivotal-voter model}

A community is holding a referendum. There are $n$ citizens, indexed by $i \in\{1, \ldots, n\}$. These citizens are divided into supporters and opposers of the proposal. Supporters obtain a benefit $b$ from the proposed change, while opposers incur a loss $x$. Each citizen knows whether he is a supporter or an opposer, but does not know the number of citizens in each category. All citizens know the probability of a randomly selected individual being a supporter is $\mu$.

Citizens must decide whether to vote in the referendum. If they do, supporters vote in favor and opposers vote against. Each citizen $i$ faces a cost of voting $c_{i}$ where $c_{i}$ is the realization of a random variable uniformly distributed on $[0, c]$. Citizens observe their own voting costs, but only know that the costs of their fellows are the independent realizations of $n-1$ random variables.

The only benefit of voting is the instrumental benefit of changing the outcome. Since the probability of being pivotal depends upon who else is voting, voting is a

5 Estimating the group rule-utilitarian model with a finite number of voters is computationally infeasible. 
strategic decision. Accordingly, the situation is modelled as a game of incomplete information in which nature chooses the number of supporters and citizens' voting costs. Then, each citizen, having observed his own voting cost, decides whether or not to vote. If the number of votes in favor of the referendum is at least as big as the number against, the proposed change is approved.

A strategy for a citizen $i$ is a function which for each possible realization of his voting cost specifies whether he will vote or abstain. The equilibrium concept is Bayesian-Nash equilibrium - each citizen must be happy with his strategy given the strategies of the other citizens and his statistical knowledge concerning the distribution of supporters and voting costs. Following Palfrey and Rosenthal (1985), we look for a symmetric equilibrium in which supporters and opposers use common strategies. With no loss of generality, we can assume that supporters and opposers use "cut-off" strategies that specify that they vote if and only if their cost of voting is below some critical level. Accordingly, a symmetric equilibrium is characterized by a pair of numbers $\gamma_{s}^{*}$ and $\gamma_{o}^{*}$ representing the cut-off cost levels of the two groups.

To characterize the equilibrium cut-off levels, consider the decision of some citizen $i$. Suppose the remaining $n-1$ citizens are playing according to the equilibrium strategies; i.e., supporters (opposers) vote if their voting cost is less than $\gamma_{s}^{*}\left(\gamma_{o}^{*}\right)$. Let $\rho\left(v_{s}, v_{o} ; \gamma_{s}^{*}, \gamma_{o}^{*}\right)$ denote the probability that $v_{s}$ of the $n-1$ individuals vote in support and $v_{o}$ vote in opposition when they play according to the equilibrium strategies. We show how to compute this below. Recall that the referendum passes if and only if at least as many people vote for as against the proposed change. Thus, if citizen $i$ is a supporter, he will be pivotal whenever $v$ of the $n-1$ other individuals vote in opposition and $v-1$ vote in support. In all other circumstances, his vote does not impact the outcome. Accordingly, the expected benefit of $i$ voting is ${ }^{6}$

$$
\sum_{v=1}^{n / 2} \rho\left(v-1, v ; \gamma_{s}^{*}, \gamma_{o}^{*}\right) b .
$$

Individual $i$ will wish to vote if this expected benefit exceeds his cost of voting.

\footnotetext{
6 This assumes that $n$ is even. The case in which $n$ is odd requires obvious modifications.
} 
Accordingly, in equilibrium

$$
\sum_{v=1}^{n / 2} \rho\left(v-1, v ; \gamma_{s}^{*}, \gamma_{o}^{*}\right) b=\gamma_{s}^{*}
$$

If citizen $i$ is an opposer, he will be pivotal whenever $v$ of the $n-1$ other individuals vote in opposition and $v$ vote in support. In all other circumstances, his vote does not impact the outcome. Accordingly, the expected benefit of $i$ voting is

$$
\sum_{v=0}^{n / 2-1} \rho\left(v, v ; \gamma_{s}^{*}, \gamma_{o}^{*}\right) x
$$

In equilibrium, we have that:

$$
\sum_{v=0}^{n / 2-1} \rho\left(v, v ; \gamma_{s}^{*}, \gamma_{o}^{*}\right) x=\gamma_{o}^{*} .
$$

Equations (2) and (4) give us two equations in the two unknown equilibrium variables $\gamma_{s}^{*}$ and $\gamma_{o}^{*}$. The exogenous parameters of the model are the probability that each individual is a supporter $\mu$, the benefit to supporters $b$, the loss to opposers $x$, and the upper bound of the cost distribution $c$. Existence of an equilibrium pair $\gamma_{s}^{*}$ and $\gamma_{o}^{*}$ for any given values of the exogenous parameters is not an issue (see Ledyard (1984) and Palfrey and Rosenthal (1985)), but there might in principle be multiple solutions. While Borgers (2004) has shown that there is a unique equilibrium when $b=x$ and $\mu=1 / 2$, there is no general uniqueness result in the literature.

To compute equilibria we need to know the function $\rho\left(v_{s}, v_{o} ; \gamma_{s}^{*}, \gamma_{o}^{*}\right)$. Let $P(s)$ denote the probability that $s$ of the $n-1$ other citizens are supporters. This is given by:

$$
P(s)=\left(\begin{array}{c}
n-1 \\
s
\end{array}\right) \mu^{s}(1-\mu)^{n-1-s} .
$$

If there are $s$ supporters, the probability that $v_{s} \in\{1, \ldots, s\}$ vote in support is

$$
\left(\begin{array}{c}
s \\
v_{s}
\end{array}\right)\left(\frac{\gamma_{s}^{*}}{c}\right)^{v_{s}}\left(1-\frac{\gamma_{s}^{*}}{c}\right)^{s-v_{s}} .
$$

Similarly, the probability that $v_{o} \in\{1, \ldots, n-1-s\}$ vote in opposition is

$$
\left(\begin{array}{c}
n-1-s \\
v_{o}
\end{array}\right)\left(\frac{\gamma_{o}^{*}}{c}\right)^{v_{o}}\left(1-\frac{\gamma_{o}^{*}}{c}\right)^{n-1-s-v_{o}} .
$$


Thus, the probability that $v_{s}$ vote in support and $v_{o}$ vote in opposition is

$$
\begin{aligned}
& \rho\left(v_{s}, v_{o} ; \gamma_{s}^{*}, \gamma_{o}^{*}\right)= \\
& \sum_{s=v_{s}}^{n-1-v_{o}}\left(\begin{array}{c}
s \\
v_{s}
\end{array}\right)\left(\frac{\gamma_{s}^{*}}{c}\right)^{v_{s}}\left(1-\frac{\gamma_{s}^{*}}{c}\right)^{s-v_{s}}\left(\begin{array}{c}
n-1-s \\
v_{o}
\end{array}\right)\left(\frac{\gamma_{o}^{*}}{c}\right)^{v_{o}}\left(1-\frac{\gamma_{o}^{*}}{c}\right)^{n-1-s-v_{o}} P(s) .
\end{aligned}
$$

Because (2) and (4) are a system of nonlinear equations, with no reduced form solution, equilibria can only be found numerically. This is a computationally difficult problem. There is no algorithm guaranteeing that $a$ solution of a system of linear equations can be found, let alone all solutions. Our approach is to use a standard root-computation routine to find a solution from a user-given starting point.

\section{Texas liquor referenda}

\subsection{Institutional background ${ }^{7}$}

Chapter 251 of the Texas Alcoholic Beverage Code states that "On proper petition by the required number of voters of a county, or of a justice precinct or incorporated city or town in the county, the Commissioners' Court shall order a local election in the political subdivision to determine whether or not the sale of alcoholic beverages of one or more of the various types and alcoholic contents shall be prohibited or legalized in the county, justice precinct, or incorporated city or town". Thus, citizens can propose changes in the liquor laws of their communities and have their proposals directly voted on in a referendum. Such direct democracy has a long history in Texas, with local liquor elections dating back to the mid-1800s.

The process by which citizens may propose a change for their jurisdiction is relatively straightforward. The first step involves applying to the Registrar of Voters for a petition. This only requires the signatures of ten or more registered voters in the jurisdiction. The hard work comes after receipt of the petition. The applicants must get it signed by at least $35 \%$ of the registered voters in their jurisdiction and must do this within thirty days. ${ }^{8}$ If this hurdle is successfully completed, the

\footnotetext{
${ }^{7}$ This discussion of the institutional details draws on Coate and Conlin (2004).

8 Prior to 1993 , the number of signatures needed was $35 \%$ of the total number of votes cast in the last preceding gubernatorial election.
} 
Commissioners' Court of the county to which the jurisdiction belongs must order a referendum be held. This order must be issued at its first regular session following the completion of the petition and the referendum must be held between twenty and thirty days from the time of the order. All registered voters can vote and if the proposed change receives at least as many affirmative as negative votes, it is approved.

Citizens may propose changes for their entire county, their justice precinct, or the city or town in which they reside. The state is divided into 254 counties and each county is divided into justice precincts. ${ }^{9}$ Accordingly, a justice precinct lies within the county to which it belongs. By contrast, a city may spillover into two or more justice precincts. If only part of a city belongs to a particular justice precinct that has approved a change, then that part must abide by the new regulations. However, if the city then subsequently approved a different set of regulations, they would also be binding on the part contained in the justice precinct in question. Effectively, current regulations are determined by the most recently approved referendum.

Importantly for our purposes, liquor referenda are typically held separately from other elections. Section 41.01 of the Texas Election Laws sets aside four dates each year as uniform election dates. These are the dates when presidential, gubernatorial, and congressional elections are held. In addition, other issues are often decided on these days such as the election of aldermen, and the approval of the sale of public land and bond issuances. Elections pertaining to these other issues may occur, but rarely do, on dates other than uniform election days. Liquor referenda, in contrast, do not typically occur on uniform election dates. This reflects the tight restrictions placed by Chapter 251 on the timing of elections. ${ }^{10}$

\subsection{Data}

Coate and Conlin (2004) assembled data on 366 local liquor elections in Texas between 1976 and 1996 where prior to the election the voting jurisdictions prohibited

\footnotetext{
9 The number of justice precincts in a county range from 1 to 8 .

10 Interestingly, the Texas state government voted in 2001 to require liquor law referendum votes to occur on one of the four uniform election dates. This was to avoid the costs of holding referenda separately.
} 


\begin{tabular}{l|c|c} 
& Small jurisdictions & Large jurisdictions \\
\hline \hline Number of referenda & 144 & 222 \\
Jurisdiction characteristics & & \\
\hline Voting age population & $370(200)$ & $6,539(8,742)$ \\
Fraction of baptists & $52 \%(11)$ & $46 \%(14)$ \\
Located in an MSA & $44 \%(50)$ & $43 \%(50)$ \\
Incorporated city or town & $95 \%(22)$ & $42 \%(50)$ \\
& & \\
Referendum characteristics & & \\
\hline Beer/wine & $46 \%(50)$ & $37 \%(48)$ \\
Off-premise & $40 \%(49)$ & $39 \%(49)$ \\
Off- and on-premise & $15 \%(35)$ & $24 \%(43)$ \\
More liberal than county & $42 \%(49)$ & $28 \%(45)$ \\
Held on weekend & $68 \%(47)$ & $72 \%(45)$ \\
Turnout & $54 \%(19)$ & $24 \%(15)$ \\
Closeness & $-1.0 \%(21)$ & $-1.9 \%(6.7)$
\end{tabular}

Table 1: Summary Statistics (standard deviations in parentheses)

the retail sale of all alcohol. The Texas Alcoholic Beverage Commission provided the details of the referenda as well as the election results. This data was augmented with information from the U.S. Census and Churches \& Church Membership in the U.S.. The voting age population in these 366 jurisdictions averaged 4,112 and ranged from 43 to 81,904. Because of computational constraints, we estimate the parameters of the pivotal-voter model using only those "small" elections where the number of citizens over age 18 is less than 900. There are 144 such referenda.

Table 1 provides summary statistics for the "within-sample" (less than 900 voting age citizens) and "out-of-sample" observations (more than 900 voting age citizens). The average number of eligible voters is 370 for the smaller jurisdictions and 6,539 for the larger jurisdictions. The fraction of baptists in the population and the fraction located in an MSA are similar across small and large jurisdictions. Small jurisdictions are more likely to be incorporated cities or towns. Those jurisdictions 


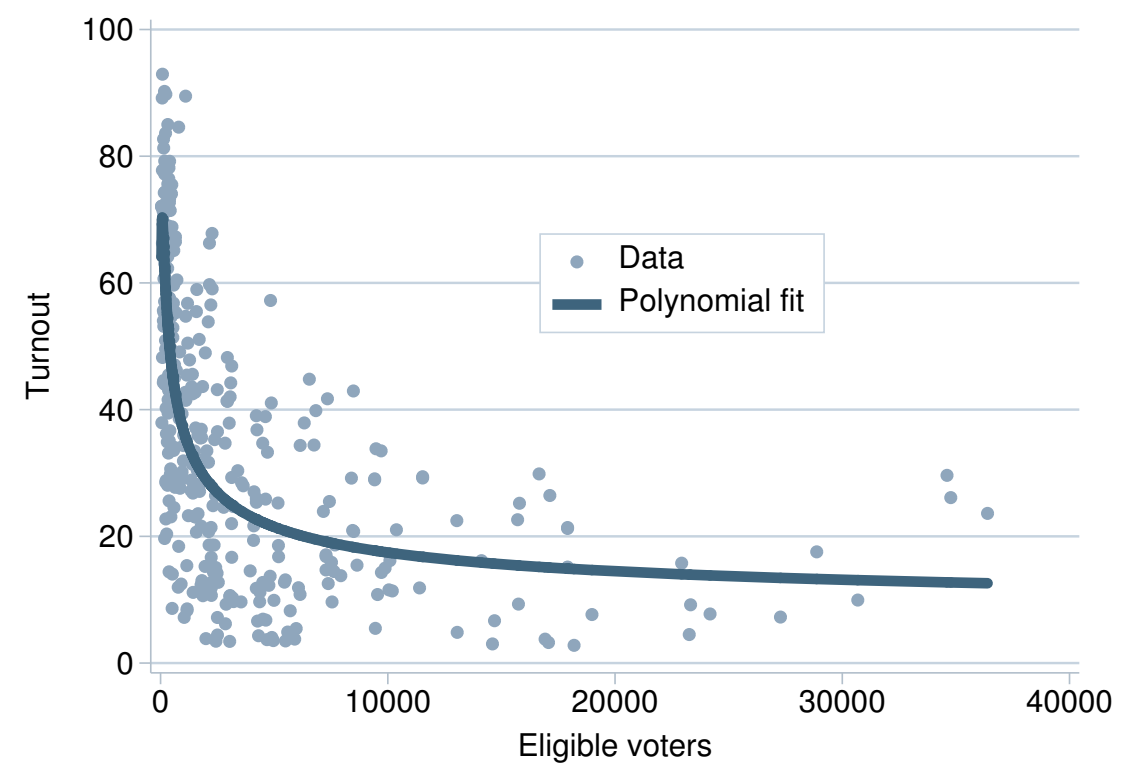

Figure 1: Voter turnout as a percent of eligible voters in the data

that are not incorporated cities or towns are almost all justice precincts, with only two referenda involving an entire county (both large jurisdictions).

While all the elections involved eliminating restrictions imposed on the sale of alcohol, they differed in the scope of reform. Beer/wine referenda permit only the sale of beer and wine. Off-premise referenda allow the sale of all liquor for consumption off-premise (i.e., no bars). Off- and on-premise referenda are the most permissive, permitting both off- and on-premise consumption of all liquor. Table 1 indicates that for both the small and large jurisdictions, the majority of referenda were either beer/wine or off-premise. Observe also that referenda in small jurisdictions are more likely to be proposing a more liberal policy than prevailing in the rest of the county. The fraction of elections held on the weekend is similar across the sets.

Table 1 indicates that turnout is substantially lower in the larger jurisdictions, $24 \%$ of eligible voters as opposed to $54 \%$. Figure 1 provides a closer look at the relationship between jurisdiction size and turnout. The scatter plot represents the 


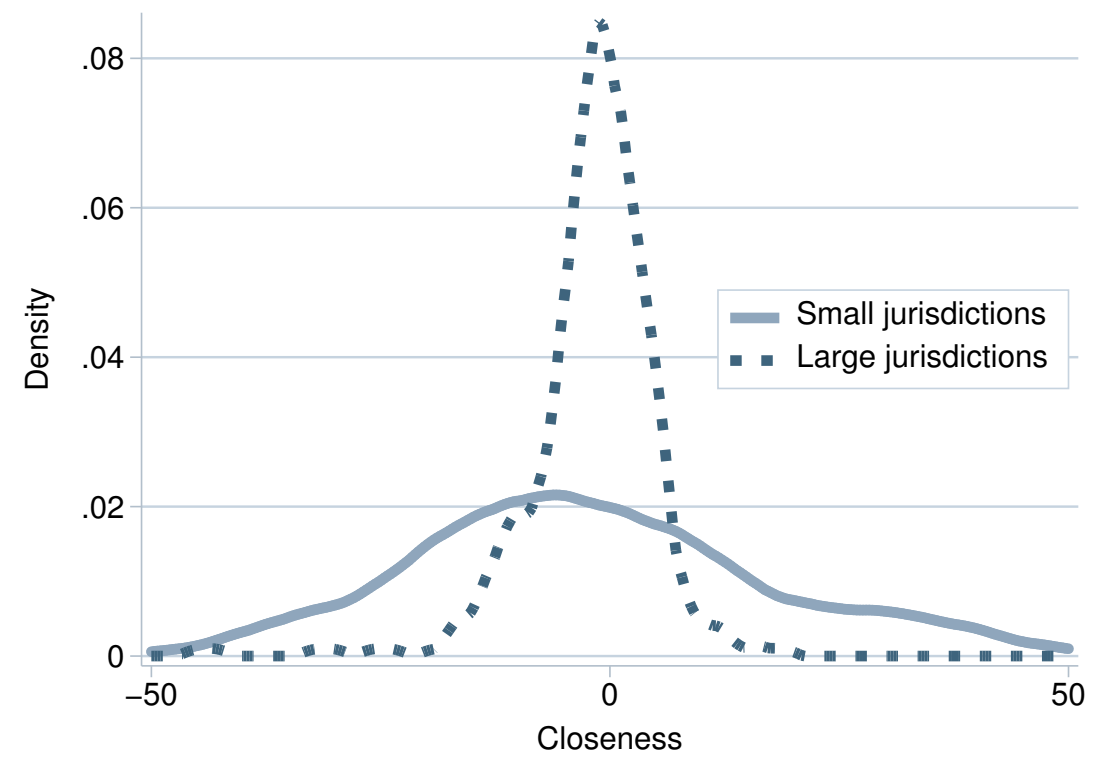

Figure 2: Distribution over vote closeness in the data

percent turnout in each jurisdiction and the dark line is a polynomial fit across these observations. ${ }^{11}$ This line indicates that turnout decreases sharply from almost $80 \%$ in small jurisdictions to about $20 \%$ as the jurisdiction size increases up to approximately 5,000 eligible voters. However, it levels off when the number of eligible voters is greater than 5,000 .

Table 1 also reports information on closeness, defined as the difference between percent turnout for and percent turnout against the proposed change. Turnout for is, on average, $1.0 \%$ less and $1.9 \%$ less than turnout against in the small and large jurisdictions, respectively. This resulted in $45 \%$ of the referenda passing in the small jurisdictions and $40 \%$ in the large jurisdictions. The standard deviations associated with these average closeness figures indicate that elections in small jurisdictions are more likely to be close. The average winning margin, defined as the absolute value of closeness is $16 \%$ in the small jurisdictions compared to $4.6 \%$ in the large jurisdictions. Figure 2 depicts, for both small and large jurisdictions, the distribution of closeness.

11 The figure omits two outliers with more than 45,000 eligible voters. 
The solid line shows the kernel density estimate of the closeness of the referendum outcomes in small jurisdictions while the dashed line is the kernel density estimate for the larger jurisdictions. Figure 2 indicates that there is considerable variation in closeness across jurisdictions and that this variation is greater for the smaller jurisdictions. ${ }^{12}$

\section{Estimation}

\subsection{Identification}

Our goal is to provide inference on the parameters of the model using the data on election outcomes in Texas liquor referenda. Before describing in detail the estimation procedure, it is important to discuss what features of the data allow us to identify the coefficients associated with the model's parameters. Recall that the model outlined in section 2 is characterized by just four parameters: the supporters' benefit $b$; the opposers' loss $x$; the probability that a citizen is a supporter $\mu$; and the upper bound of the uniform cost distribution, $c$. We will consider how changes in the values of the parameters impact the outcome predicted by the model.

To simplify the discussion, assume that all jurisdictions share the same values of $b, x, \mu$, and $c$. Note first that only the relative values of $b, x$, and $c$ matter; that is, when $b, x$, and $c$ are multiplied by the same factor, the equilibrium values of $\gamma_{s}$ and $\gamma_{o}$ are also scaled by the same factor, and the probability of observing a referendum outcome (the number of votes for and against) conditional on the equilibrium cut-off levels is independent of the scaling factor. Therefore, under the simplifying assumption that all jurisdictions are identical, $c$ is not identified, and we can normalize $c$ to 1 .

Next observe that the combined magnitude of parameters $b$ and $x$ effects overall turnout, because increasing the benefits from winning the election increases the incentives to vote, and therefore turnout. What is left is the determination of the difference $b-x$ and $\mu$. Both $b-x$ and $\mu$ are identified from the observed difference

12 This pattern remains when the jurisdictions are broken into smaller subsamples based on number of eligible voters. 
between turnout for and turnout against. We are able to separately identify $b-x$ and $\mu$ because: (i) a change in $b-x$ has different effects than a change in $\mu$ on turnout; and (ii) the differential effects of changes in $b-x$ and $\mu$ vary with the size of the jurisdiction and total turnout. For example, the difference between votes for and against provides more information on $\mu$ relative to $b-x$ when a jurisdiction has high total turnout and a large number of eligible voters. Hence, variation in closeness of the electoral outcome across jurisdictions with different levels of turnout and different numbers of eligible voters facilitate the identification of $\mu$ from $b-x$.

We conclude that, under the assumption that all jurisdictions have identical $b$, $x, \mu$, and $c$, identification of these parameters is possible (after normalizing the cost of voting) provided that sufficient variation exists across jurisdictions in eligible voters and referendum outcome. In our empirical implementation, we allow the four parameters to have different values by having them depend on exogenous jurisdiction characteristics. The coefficients associated with these jurisdiction specific variables are identified from the variation in electoral outcomes across jurisdictions with different exogenous characteristics.

\subsection{Estimation procedure}

We assume that each of our 144 jurisdictions is characterized by a distinct quadruple of parameters $\left(\mu_{j}, b_{j}, x_{j}, c_{j}\right)$ but that each parameter is determined by jurisdiction and referenda specific characteristics in a common way. Specifically, for each jurisdiction $j$, we assume that:

$$
\begin{aligned}
b_{j} & =\exp \left(\boldsymbol{\beta}^{b} \cdot \mathbf{z}_{j}^{b}\right) \\
x_{j} & =\exp \left(\boldsymbol{\beta}^{x} \cdot \mathbf{z}_{j}^{x}\right) \\
\mu_{j} & =\frac{\exp \left(\boldsymbol{\beta}^{\mu} \cdot \mathbf{z}_{j}^{\mu}\right)}{1+\exp \left(\boldsymbol{\beta}^{\mu} \cdot \mathbf{z}_{j}^{\mu}\right)} \\
c_{j} & =\exp \left(\beta^{c} \cdot z_{j}^{c}\right)
\end{aligned}
$$

where $\left(\boldsymbol{\beta}^{b}, \boldsymbol{\beta}^{x}, \boldsymbol{\beta}^{\mu}, \beta^{c}\right)$ are vectors of coefficients to be determined and $\left(\mathbf{z}_{j}^{b}, \mathbf{z}_{j}^{x}, \mathbf{z}_{j}^{\mu}, z_{j}^{c}\right)$ are vectors of observable jurisdiction and referenda characteristics. More specifically, $\mathbf{z}_{j}^{b}=\mathbf{z}_{j}^{x}=(1$, off-premise, off- and on-premise, incorporated city or town, more liberal 
than county), $\mathbf{z}_{j}^{\mu}=\left(1\right.$, fraction baptist, MSA), and $z_{j}^{c}=($ election on weekend $)$. The functional forms for $b_{j}, x_{j}$ and $c_{j}$ are selected so that they are positive and the functional form for $\mu_{j}$ is selected to ensure that it lies between zero and one. The formulation also embodies the normalization that $c_{j}$ equals 1 when the election is not held on the weekend.

The task is to estimate the coefficients $\left(\boldsymbol{\beta}^{b}, \boldsymbol{\beta}^{x}, \boldsymbol{\beta}^{\mu}, \beta^{c}\right)$. For each jurisdiction $j$ we observe the votes for and against the proposed change, together with the total number of eligible voters which we denote respectively as $v_{s j}, v_{o j}$, and $n_{j}$. The choice of $\left(\boldsymbol{\beta}^{b}, \boldsymbol{\beta}^{x}, \boldsymbol{\beta}^{\mu}, \beta^{c}\right)$ determine $b_{j}, x_{j}, \mu_{j}$ and $c_{j}$ and these determine via equations (2) and (4) a set of $M_{j}$ equilibria in each jurisdiction $j$, which we denote $\left\{\gamma_{s j}^{m}, \gamma_{o j}^{m}\right\}_{m=1}^{M_{j}}$.

Each equilibrium implies a probability distribution over election outcomes. In particular, the probability of observing data $\left(v_{s j}, v_{o j}\right)$ conditional on the citizens voting according to the cut-off levels $\left(\gamma_{s j}, \gamma_{o j}\right)$ is given by (8). While it is impossible to know a priori whether there are multiple equilibria, if there is multiplicity we need to ensure that there is a unique mapping from the parameter space to the likelihood of observable events. We resolve this issue by assuming that the equilibrium selected is the first equilibrium the computation routine finds, using as a starting point of the routine the midpoint of the strategy space. ${ }^{13}$

Assuming the above equilibrium selection rule, denote the selected equilibrium $\left(\gamma_{s j}^{m *}, \gamma_{o j}^{m *}\right)$. Then, equation (8) defines the likelihood of observing a referendum outcome conditional on the equilibrium cut-off levels $\left(\gamma_{s j}^{m *}, \gamma_{o j}^{m *}\right)$. The likelihood function is therefore

$$
L\left(\boldsymbol{\beta}^{b}, \boldsymbol{\beta}^{x}, \boldsymbol{\beta}^{\mu}, \beta^{c}\right)=\prod_{j} \rho\left(v_{s j}, v_{o j} ; \gamma_{s j}^{m *}, \gamma_{o j}^{m *}\right) .
$$

The estimation strategy is the following: first, guess a vector of coefficient values $\left(\boldsymbol{\beta}^{b}\right.$, $\left.\boldsymbol{\beta}^{x}, \boldsymbol{\beta}^{\mu}, \beta^{c}\right)$. Then compute equilibrium critical cost levels for each jurisdiction (based

13 An alternative procedure is to assume an equilibrium selection rule, and estimate the parameters of the equilibrium selection rule together with the parameters of the model. We experimented with this procedure, but found it computationally unfeasible. The procedure we chose is arbitrary only to the extent in which multiplicity is prevalent. We investigated this issue and found that multiplicity does not arise very often. We thoroughly searched for equilibria using a grid of parameter vectors centered around our estimates. For all of these parameter vectors, we found multiplicity in less than ten percent of the jurisdictions. At the estimated values of the parameters, we found only 7 jurisdictions displaying multiple equilibria. 
on the equilibrium selection rule stated above), and the probability of observing the jurisdiction election outcome conditional on such thresholds. These probabilities determine the likelihood of observing the data given by $L\left(\boldsymbol{\beta}^{b}, \boldsymbol{\beta}^{x}, \boldsymbol{\beta}^{\mu}, \beta^{c}\right)$. Finally, find the coefficient values that maximize $L\left(\boldsymbol{\beta}^{b}, \boldsymbol{\beta}^{x}, \boldsymbol{\beta}^{\mu}, \beta^{c}\right)$.

The sums in equations (2), (4), and (8) take progressively longer to compute as the number of eligible voters increases, making the computation of the equilibria extremely time consuming in large jurisdictions, even using Normal approximations to the Binomial distributions. This explains why we limited our sample to the 144 observations with the smallest number of eligible voters. ${ }^{14}$

\section{Results}

\subsection{Coefficient estimates}

Table 2 contains the coefficient values that maximize the likelihood function, with bootstrapped standard errors in parentheses, while Table 3 provides the average values of the model's exogenous variables implied by these parameter estimates.

The point estimates in Table 2 suggest that the probability an individual is a supporter does not depend appreciably on the fraction of baptists in the jurisdiction or on whether the jurisdiction is located in an MSA. The coefficient estimates associated with the benefit and loss parameters, $b$ and $x$, suggest that the effect of the jurisdiction-specific characteristics on preferences are often quite large and similar for supporters and opposers. Both supporters and opposers benefit more from their preferred outcome if the jurisdiction holding the election is an incorporated city or town. Less intuitively, both groups' benefits are less for off- and on-premise referenda compared to off-premise referenda and beer/wine referenda (the omitted category). The average supporter's benefit and opposer's loss almost double when the jurisdiction is a city/town and are approximately fifty percent less for an offand on-premise referendum than a beer/wine referendum. The benefit and loss parameters increase slightly if the referendum passing results in the jurisdiction having

14 We used an implementation of the simulated annealing method developed by Bill Goffe (see Goffe et al. (1992)) to maximize (13) on a 16-processor supercomputer. 


\begin{tabular}{cl|c|c}
\multicolumn{2}{c|}{ Parameter/Variable $(\ln L:-5694.21)$} & Estimate & Marginal Effect \\
\hline \hline$\mu: \quad$ Fraction of baptists & $-0.058(0.188)$ & -0.015 \\
& Located in an MSA & $-0.089(0.072)$ & -0.022 \\
Constant & $0.062(0.097)$ & \\
\hline$b: \quad$ Off-premise & $0.182(0.086)$ & 2.85 \\
& Off- and on-premise & $-0.642(0.232)$ & -7.89 \\
& Incorporated city or town & $1.819(0.354)$ & 13.68 \\
& More liberal than county & $0.199(0.068)$ & 3.15 \\
Constant & $0.875(0.405)$ & \\
\hline$x: \quad$ Off-premise consumption & $0.097(0.082)$ & 1.56 \\
$\quad$ Off- and on-premise & $-0.589(0.253)$ & -7.58 \\
& Incorporated city or town & $1.791(0.340)$ & 13.97 \\
More liberal than county & $0.361(0.062)$ & 5.90 \\
Constant & $0.886(0.370)$ & \\
\hline$c: \quad$ Held on weekend & $-0.172(0.085)$ & -0.16
\end{tabular}

Table 2: Pivotal voter model estimates (standard errors in parentheses) ${ }^{15}$

a more liberal alcohol policy than in the rest of the county. In terms of the cost of voting, the coefficient estimates suggest that having an election on a weekend decreases average voting costs by approximately 16 percent.

The average values of the model's exogenous variables implied by the parameter estimates (Table 3) indicate that, on average, citizens are evenly divided between supporters and opposers (i.e., $\mu=.50$ ). The average benefit of supporters is slightly less than the loss of opposers, which means that the average probability a supporter votes is slightly less than that of an opposer (0.516 versus 0.530$)$. These probabilities are quite large because both the average benefit to supporters and the loss to opposers are approximately 35 times greater than the average voting costs.

The implied values of the model's exogenous variables (Table 3) can be interpreted in dollar terms if we assign a value to the cost of voting. For example, suppose

\footnotetext{
${ }^{15}$ For the "Fraction of baptists" variable, the marginal effect column reports the percent change in the value of parameter $\mu$ given a one percent change in the fraction of baptists. For all other variables, which are all dummies, the entry is the percent change in the value of the parameter with respect to the change from zero to one in the independent variable.
} 


\begin{tabular}{l|c}
\multicolumn{1}{c|}{ Parameter } & Mean estimate \\
\hline \hline Fraction of supporters $\mu$ & $0.500(0.011)$ \\
Supporters' benefit $b$ & $15.52(4.81)$ \\
Opposers' benefit $x$ & $15.90(5.12)$ \\
Upper bound on cost $c$ & $0.892(0.074)$ \\
Supporters that vote $\frac{\gamma_{s}}{c}$ & $0.516(0.167)$ \\
Opposers that vote $\frac{\gamma_{o}}{c}$ & $0.530(0.174)$
\end{tabular}

Table 3: Mean parameter estimates (standard errors in parentheses)

that we guess that the average voting cost across all jurisdictions is $\$ 10$. Then, this implies that $c / 2=0.892 / 2=\$ 10$. This ties down the units in dollar terms since $0.892=\$ 20$ or $1=\$ 20 /(0.892)=\$ 22.42$. It follows that the average value of $b$ is $\$ 22.42(15.52)=\$ 347.98$ and the average value of $x$ is $\$ 22.42(15.90)=\$ 356.50$. These numbers are on the large side, but within the range of plausibility.

\subsection{Model performance}

We begin with the within-sample fit of the model. Two distinct dimensions of model performance may be identified. First, how well does the model predict total turnout? Second, how well does the model predict the closeness of the election, that is, the difference between turnout for and turnout against the proposed change?

To obtain the predicted voting behavior, we use the coefficient estimates in Table 2 to calculate the implied values of the model's parameters $\left(b_{j}, x_{j}, \mu_{j}, c_{j}\right)$ for each jurisdiction and compute a pair of cut-off levels $\left(\gamma_{s j}^{*}, \gamma_{o j}^{*}\right)$ that satisfies the equilibrium conditions (2) and (4). Then we simulate election outcomes for all 144 jurisdictions. We do this by letting the computer draw for each jurisdiction a particular realization of preferences and cost shocks from the distribution associated with that jurisdiction. Thus, in jurisdiction $j$, the probability that a citizen is a supporter is $\mu_{j}$ and each citizen's voting cost is drawn from the uniform distribution on $\left[0, c_{j}\right]$. These draws together with the equilibrium cut-offs then determine turnout for and against the proposed change. Each (aggregate) draw determines a sample with a pattern 


\begin{tabular}{c|c|c|c} 
Eligible voters $n$ & N. of obs. & Data & $\begin{array}{c}\text { Pivotal-voter } \\
\text { model }\end{array}$ \\
\hline \hline$n<247$ & 48 & 0.62 & 0.65 \\
$247<n<434$ & 48 & 0.55 & 0.51 \\
$434<n<900$ & 48 & 0.43 & 0.40 \\
All within-sample $(n<900)$ & 144 & 0.54 & 0.52
\end{tabular}

Table 4: Average turnout as a percentage of eligible voters: model vs. data

of election outcomes across the jurisdictions. By repeating the procedure numerous times and averaging across all samples we can compute the predicted distributions for total turnout and closeness implied by the model.

Table 4 compares average actual turnout with the average predicted from the simulations. It does this for all within-sample observations and for various smaller subsamples based on jurisdiction size. The table indicates that the pivotal-voter model is relatively accurate in predicting the average total turnout of 0.54 for all within-sample observations as well as the average turnouts for the equally-sized subsamples.

The performance of the model in predicting the electoral outcomes of out-ofsample jurisdictions is worse. To obtain the predicted voting behavior for the out-ofsample jurisdictions, we assume the coefficient estimates in Table 2 apply and again compute a pair of critical cost levels that satisfies the equilibrium conditions. ${ }^{16}$ We then calculate the predicted distributions over turnout. Unlike the withinsample comparison, the model significantly under-predicts turnout for the large jurisdictions. The average actual turnout in these out-of-sample observations is 0.24 while the pivotal-voter model predicts an average of 0.14 . This is perhaps not surprising in light of the theoretical result from Palfrey and Rosenthal (1984) that we cite in the introduction.

\footnotetext{
16 This procedure assumes that the coefficients estimated for the smaller jurisdictions are valid for the larger jurisdictions.
} 


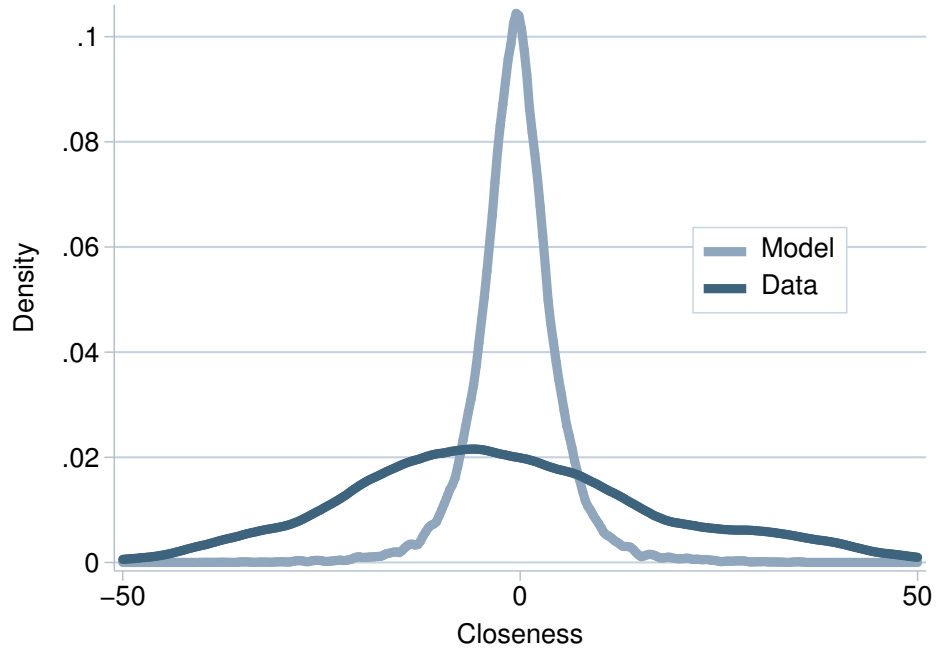

Figure 3: Distributions over closeness, model vs. data (within-sample)

Turning to closeness, Figure 3 depicts the distributions of actual and predicted closeness obtained from our simulations. The darker line is the predicted kernel density estimate of the distribution from the model, while the lighter line is a kernel density estimate of the distribution in the data. It is evident from Figure 3 that the model predicts much closer elections than are observed in the data. This seems likely to reflect the basic logic of the model - if an election were not expected to be close, then the probability of being pivotal would be very low and there would be little incentive to vote. However, another possible explanation involves jurisdictionspecific heterogeneity not accounted for by our set of covariates. This unobserved heterogeneity makes us reluctant to use the evidence on closeness alone to declare the pivotal-voter model unsatisfactory. Therefore, in the next section we compare the performance of the pivotal-voter model to that of a simple alternative model, which we estimate using the same set of explanatory variables. 


\section{A simple alternative model}

While the pivotal-voter model yields reasonable coefficient estimates and fits the pattern of turnout within-sample reasonably well, it predicts that elections will be much closer than they actually are. It is therefore natural to wonder whether a simpler model based on the idea of citizens voting for non-instrumental reasons might fit the data better. To investigate this we study the comparative performance of a simple expressive model of voting.

The expressive view asserts that citizens vote not to impact the outcome but to express their preferences (see, for example, Brennan and Lomasky (1993)). It seems natural to assume that people care more about expressing their preferences the more intensely they feel about an issue. This suggests what Coate and Conlin (2004) refer to as the intensity model which works within the same basic environment as the pivotal-voter model but assumes that supporters vote if their voting cost is less than $\gamma_{s}=\alpha b$, while opposers vote if their voting cost is less than $\gamma_{o}=\alpha x$, where $\alpha>0$. Here, the parameter $\alpha$ measures the strength of citizens' desire to express themselves through voting. The key restriction is that both supporters and opposers share the same $\alpha$. Under this specification, the probability that a supporter votes is the probability that $\gamma_{s}$ exceeds his voting cost, which is $\gamma_{s} / c=\alpha b / c$. Similarly, the probability that an opposer votes is $\gamma_{o} / c=\alpha x / c$. Note that, unlike the pivotalvoter model, the propensity of a supporter (opposer) to vote does not depend on the propensity of an opposer (supporter) to vote.

We assume that the parameters $b_{j}, x_{j}, \mu_{j}$, and $c_{j}$ depend on the same jurisdiction characteristics as in the pivotal-voter model (see equations 9-12). In addition, we assume that:

$$
\alpha_{j}=(\text { number of eligible voters in jurisdiction } j)^{\beta}
$$

Thus, we allow citizens' desire to express themselves to vary with the size of their community. Observe that our specification for $\alpha_{j}$ contains no constant term. This normalization reflects the fact that we cannot separately identify $\alpha_{j}, b_{j}$ and $x_{j}$. The

effect on voting behavior of an increase in the desire of citizens to express themselves (i.e., an increase in $\alpha_{j}$ ) can be mimicked by an increase in how strongly they feel 


\begin{tabular}{ll|c|c}
\multicolumn{2}{c|}{ Parameter / Variable $(\ln L:-4298.0)$} & Estimate & Marginal Effect \\
\hline \hline$\mu: \quad$ Fraction of baptists & $-0.647(0.128)$ & -0.157 \\
& Located in an MSA & $-0.362(0.025)$ & -0.088 \\
Constant & $0.180(0.095)$ & \\
\hline$b: \quad$ Off-premise & $0.163(0.019)$ & 0.406 \\
& Off- and on-premise & $-0.305(0.032)$ & -0.670 \\
& Incorporated city or town & $0.504(0.039)$ & 0.992 \\
& More liberal than county & $0.057(0.018)$ & 0.141 \\
& Constant & $0.359(0.069)$ & \\
\hline$x: \quad$ Off-premise & $0.554(0.015)$ & 0.118 \\
& Off- and on-premise & $-0.631(0.032)$ & -1.065 \\
& Incorporated city or town & $0.032(0.031)$ & 0.067 \\
& More liberal than county & $0.247(0.016)$ & 0.532 \\
& Constant & $0.659(0.092)$ & \\
\hline$c: \quad$ Held on weekend & $0.007(0.012)$ & 0.007 \\
\hline$\alpha: \quad$ Eligible voters & $-0.252(0.010)$ & -7.62
\end{tabular}

\section{Table 5: Intensity model estimates (standard errors in parenthesis) ${ }^{17}$}

about the issue (i.e., a simultaneous increase in $b_{j}$ and $x_{j}$ ). Accordingly, we can only identify the aggregate expressive benefits to supporters and opposers which are $\alpha_{j} b_{j}$ and $\alpha_{j} x_{j}$. This is not problematic given that our primary goal is to compare the fit of this model with that of the pivotal-voter model.

The task is to estimate the coefficients $\left(\boldsymbol{\beta}^{b}, \boldsymbol{\beta}^{x}, \boldsymbol{\beta}^{\mu}, \beta^{c}, \beta\right)$ and the estimation procedure is similar to that for the pivotal-voter model. The choice of coefficients determines $\alpha_{j} b_{j}, \alpha_{j} x_{j}, c_{j}$ and $\mu_{j}$ and these determine the cut-offs $\left(\gamma_{s j}=\alpha_{j} b_{j}\right.$, $\left.\gamma_{o j}=\alpha_{j} x_{j}\right)$. The probability of observing data $\left(v_{s j}, v_{o j}\right)$ conditional on citizens voting according to the cut-offs $\left(\gamma_{s j}, \gamma_{o j}\right)$ is given by (8). The likelihood function is then $L(\Omega)=\Pi_{j} \rho\left(v_{s j}, v_{o j} ; \gamma_{s j}, \gamma_{o j}\right)$ and the estimation strategy is as before.

\footnotetext{
17 For the "Fraction of baptists" variable, the marginal effect column reports the percent change in the value of parameter $\mu$ given a one percent change in the fraction of baptists. For the "Eligible voters" variable, the marginal effect column reports the percent change in the value of parameter $\alpha$ given an increase in eligible voters of 100 . For all other variables, which are all dummies, the entry is the percent change in the value of the parameter with respect to the change from zero to one in
} 


\begin{tabular}{l|c}
\multicolumn{1}{c|}{ Parameter } & Mean estimate \\
\hline \hline Fraction of supporters $\mu$ & $0.423(0.043)$ \\
Supporters' expressive benefit $\alpha b$ & $0.585(0.138)$ \\
Opposers' expressive benefit $\alpha x$ & $0.504(0.137)$ \\
Upper bound on cost $c$ & $1.005(0.003)$ \\
Supporters that vote $\frac{\gamma_{s}}{c}$ & $0.583(0.138)$ \\
Opposers that vote $\frac{\gamma_{o}}{c}$ & $0.501(0.137)$
\end{tabular}

\section{Table 6: Mean parameter estimates}

Table 5 contains the coefficient estimates that maximize this likelihood function using the 144 smaller jurisdictions. In general, these are as expected given the results in Coate and Conlin (2004) and are similar to those for the pivotal-voter model. ${ }^{18}$ The estimate associated with baptists implies that the probability of being a supporter is 15.7 percentage points less if the individual is baptist. The coefficient associated with the MSA variable suggests that being in an MSA decreases the probability an individual is a supporter by 8.8 percentage points. The coefficients associated with the $b$ and $x$ parameters indicate that individuals feel more strongly about the referendum if: (i) it is an off-premise referendum (as opposed to beer/wine); (ii) if the jurisdiction is an incorporated city or town; and (iii) if it results in the jurisdiction having the most liberal alcohol policy in the county. Individuals feel less strongly about off- and on-premise referenda. The negative coefficient associated with the eligible voters implies that increasing the number of eligible voters by 100 in a jurisdiction decreases the desire citizens have to express themselves by, on average, 7.6 percent.

Table 6 uses the coefficient estimates to present the average implied values of the model's exogenous variables. The probability of an eligible voter being a sup-

the independent variable.

18 Coate and Conlin (2004) estimate the intensity model using the full data set, but assume a continuum of voters. The continuum assumption makes votes for and against deterministic and hence it is necessary to introduce some uncertainty into the model for estimation purposes. This is done by assuming that the fraction of supporters is a draw from a Beta distribution and that there is unobserved district specific heterogeneity in the willingness to pay of supporters and opposers. 


\begin{tabular}{c|c|c|c} 
Eligible voters $n$ & N of Obs. & Data & $\begin{array}{c}\text { Intensity } \\
\text { model }\end{array}$ \\
\hline \hline$n<247$ & 48 & 0.62 & 0.63 \\
$247<n<434$ & 48 & 0.55 & 0.53 \\
$434<n<900$ & 48 & 0.43 & 0.45 \\
All within-sample $(n<900)$ & 144 & 0.54 & 0.54
\end{tabular}

Table 7: Average turnout as a percentage of eligible voters: intensity model vs. data

porter averages 0.423 across the 144 jurisdictions which is less than predicted by the pivotal-voter model. As with the pivotal-voter model, the implied probabilities of a supporter and opposer voting are both slightly over a half.

While the coefficient estimates and implied average values are of some interest, we are most concerned with the relative performance of the intensity and pivotalvoter models. To statistically test their relative performance, first note that the maximized log-likelihood value is $-4,298$ for the intensity model compared to $-5,694$ for the pivotal-voter model. ${ }^{19}$ Using the likelihood-ratio based statistic for nonnested models proposed by Vuong (1989), we can test the null hypothesis that the two models are equally close to the true data generating process against the alternative hypothesis that the intensity model is closer. ${ }^{20}$ Vuong's test statistic for this null hypothesis is 1.88 . Because this statistic has a standard normal distribution if the two models are equivalent, the null hypothesis can be rejected at the ten percent significance level.

The predictive power of the two models is also different. Table 7 is analogous to Table 4. It demonstrates that, like the pivotal-voter model, the intensity model does well in predicting average total turnout. Figure 4 allows a comparison of

19 The maximum log-likelihood value for the intensity model is $-4,567$ when $\alpha$ is constant.

20 The test statistic is calculated by taking the difference between the maximum log-likelihood values of the models and dividing by the product of the standard deviation of the difference in the log likelihood value for each observation and the square root of the number of observations. 


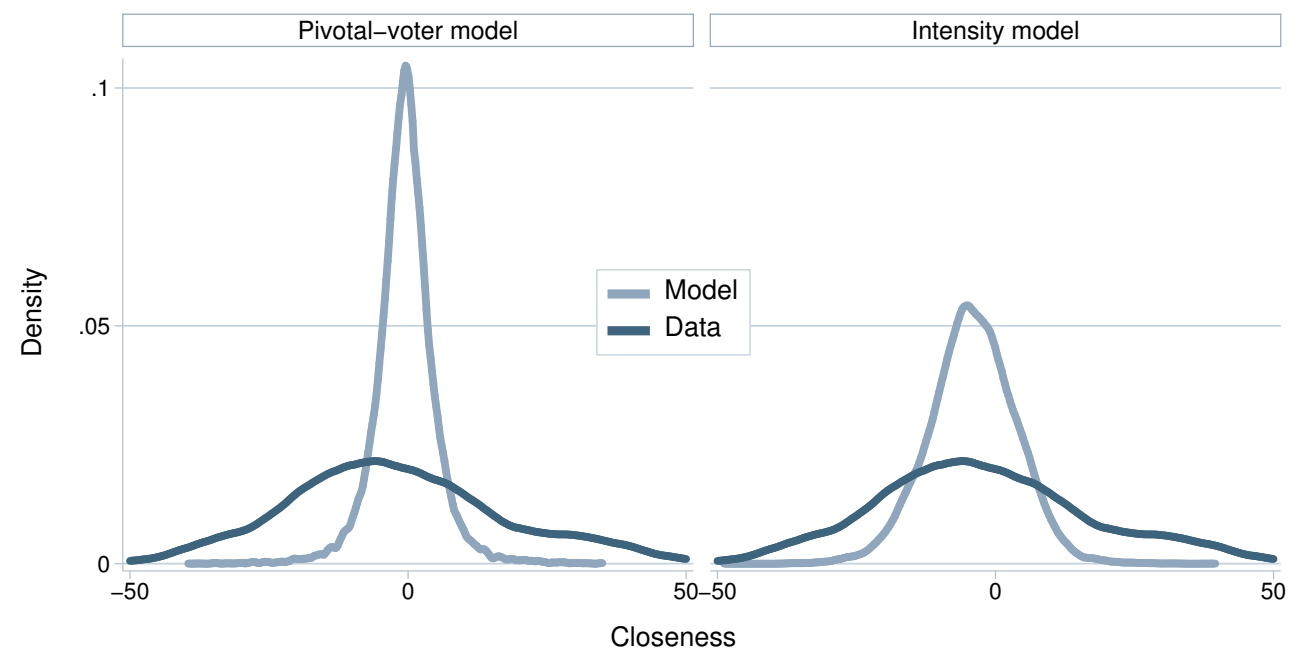

Figure 4: Distributions over closeness, pivotal and intensity model vs data (within-sample)

the relative performance of the intensity and pivotal-voter models in regards to predicting closeness. The left panel reproduces Figure 3, while the right panel is the analogous figure constructed using the simulations from the intensity model. While the intensity model predicts closer elections than are observed in the data, it does a better job than the pivotal-voter model at predicting the distribution of actual vote differences. Because the intensity model is not driven by the instrumental desire to impact the outcome, the model can generate large vote differences without jeopardizing turnout.

We also looked at the relative performance of the models in subsamples of the data formed according to the number of eligible voters. We found that the performance of the pivotal-voter model worsens as the number of eligible voters increases. Simulations on the out-of-sample observations indicate that the pivotal-voter model predicts a much more concentrated distribution of closeness than that depicted in Figure 4. The performance of the intensity model remains comparable to that displayed in Figure $4 .^{21}$

21 We also computed closeness as the supporters' vote share of total turnout. All of the results 


\section{Conclusion}

The pivotal-voter model forms the basic framework for thinking about turnout in theoretical political science. While it seems widely conceded that the model does not provide an empirically satisfactory theory of turnout in large-scale, single-issue elections, the hope has remained that it might explain voter behavior in small-scale elections. It is this hope that seems to implicitly justify the continued use of the model in theoretical work.

The results of this paper provide little to nurture this hope. While the pivotalvoter model can explain turnout in small-scale Texas liquor elections, it has difficulty explaining the large winning margins that are common in these elections. The logic of the pivotal-voter model implies that elections must be close even if there is a significant difference between the sizes of the two competing groups or the intensity of their preferences. This difficulty in explaining large winning margins allows the pivotal-voter model to be outperformed by the intensity model - a simple expressive voting model which just assumes that people are more likely to vote the more intensely they feel about the issue.

Using the same data set, Coate and Conlin (2004) have shown that a model of voter turnout in which individuals are motivated to vote by ethical reasons, fits the data well and outperforms the intensity model. Transitivity does not strictly speaking apply, because Coate and Conlin's analysis makes different assumptions, most notably a continuum of voters. Nonetheless, the combined results of the two papers certainly suggest that an ethical approach is a better way of understanding voter behavior than the pivotal-voter model. In our view, this reinforces the case for further development of the ethical approach.

\section{References}

[1] Borgers, Tilman, [2004] "Costly Voting," American Economic Review, 94(1), $57-66$

are similar to the results obtained using the measure of closeness used in the paper. Details are available from the authors upon request. 
[2] Brennan, Geoffrey and Loren Lomasky, [1993], Democracy and Decision: The Pure Theory of Electoral Preference, Cambridge: Cambridge University Press.

[3] Campbell, Colin, [1999], "Large Electorates and Decisive Minorities," Journal of Political Economy, 107(6), 1199-1217.

[4] Coate, Stephen and Michael Conlin, [2004], "A Group Rule-Utilitarian Approach to Voter Turnout: Theory and Evidence," American Economic Review, forthcoming.

[5] Feddersen, Timothy J., [2004], "Rational Choice Theory and the Paradox of Not Voting," Journal of Economic Perspectives, 18(1), 99-112.

[6] Feddersen, Timothy J. and Alvaro Sandroni, [2002], "A Theory of Participation in Elections," mimeo, Northwestern University.

[7] Ghosal, Sayantan and Ben Lockwood, [2003], "Information Aggregation, Costly Voting and Common Values," mimeo, University of Warwick.

[8] Goffe, William L., Ferrier, Gary D and Rogers, John [1992], "Simulated Annealing: An Initial Application in Econometrics," Computer Science in Economics \& Management, 5(2), 133-46.

[9] Green, Donald and Ian Shapiro, [1994], Pathologies of Rational Choice Theory: A Critique of Applications in Political Science, New Haven: Yale University Press.

[10] Hansen, Stephen; Palfrey, Thomas and Howard Rosenthal, [1987], "The Downsian Model of Electoral Participation: Formal Theory and Empirical Analysis of the Constituency Size Effect," Public Choice, 52, 15-33.

[11] Harsanyi, John C., [1980], "Rule Utilitarianism, Rights, Obligations and the Theory of Rational Behavior," Theory and Decision, 12, 115-33.

[12] Ledyard, John O., [1984], "The Pure Theory of Large Two-Candidate Elections," Public Choice, 44, 7-41. 
[13] Palfrey, Thomas and Howard Rosenthal, [1983], "A Strategic Calculus of Voting," Public Choice, 41, 7-53.

[14] Palfrey, Thomas and Howard Rosenthal, [1985], "Voter Participation and Strategic Uncertainty," American Political Science Review, 79, 62-78.

[15] Vuong, Quang H., [1989], "Likelihood Ratio Tests for Model Selection and Non-nested Hypotheses," Econometrica, 57, 307-333. 